\title{
Les enfants juifs cachés en France pendant la Seconde Guerre mondiale: des traces du traumatisme repérables plus de soixante ans après
}

\author{
Marion Feldman ${ }^{1}$, Yoram Mouchenik ${ }^{2}$, Marie Rose Moro ${ }^{3}$
}

Cette étude montre qu'il est possible d'évaluer chez des adultes, âgés entre soixante-cinq et quatre-vingt ans, les effets psychiques d'événements traumatiques vécus il y a plus de soixante ans, c'est-à-dire ayant eu lieu entre 1940 et 1945 , quand ces mêmes personnes étaient enfants.

II est question ici des enfants contraints à être cachés, afin d'être soustrait au programme d'extermination des Juifs, pendant la Seconde Guerre mondiale.

Pour être sauvé, pour échapper à la mort, les enfants juifs ont été dépendants des adultes : pas uniquement de leurs parents qui subissaient la même menace et qui ne pouvaient pas les protéger. Ces enfants ont dû parfois changer d'identité, ont connu des ruptures, des privations, ont dû quitter leur environnement familier et leurs parents. Ils ont parfois été cibles d'humiliations, de maltraitances. Ces enfants ont été transplantés brutalement, pour être protégés, mais souvent sans mots ni de la part des parents, ni de la part des adultes en charge de ces enfants. Ils recevaient uniquement des consignes pour se comporter prématurément comme des adultes: ne pas dire son nom, faire face à un danger qu'ils ne pouvaient même pas nommer, devenir des enfants invisibles.

Ces événements cumulatifs fabriquent du traumatisme (Terr, 1991). Ils ont un impact sur le développement de l'enfant, sur sa construction psychique.

Dans l'immédiat après-guerre, la souffrance de ces enfants n'a pas été reconnue : ni par les familles, ni par le collectif. Les enfants ont été "ballottés". Certains ont retrouvé leurs deux parents, d'autres l'un des deux; certains sont devenus orphelins. Parfois, ces orphelins retrouvaient des membres de leur famille (fratrie, cousins, oncles ou tantes). En plus d'avoir été cachés, ils sont devenus après la guerre, enfants de survivants de la Shoah, et/ou orphelins. Aux traumatismes vécus entre 1940 et 1945, s'ajoutent ceux de l'après-guerre.

\section{Méthodologie}

Trente-cinq entretiens de recherche ont été réalisés. Les rencontres se sont réalisées par le biais de l'association: "Enfants cachés: 1940-1944" auprès d'"enfants cachés" et par le biais d'un réseau plus informel. Ces personnes nous ont

\footnotetext{
${ }^{1}$ Docteur en psychologie clinique et psychopathologie, psychologue-clinicienne.

${ }^{2}$ HDR, maître de conférences à l'Université le Mirail / Toulouse, psychologue-clinicien

${ }^{3}$ Professeur de psychiatrie de l'enfant et de l'adolescent, Psychanalyste, Hôpital Avicenne (Bobigny), Université Paris 13 / Villetaneuse. www.clinique-transculturelle.org
} 
été adressées par notre entourage proche, par un effet de "bouche-à-oreille", ou par des rencontres dans le cadre professionnel.

De ces trente-cinq personnes rencontrées, vingt-neuf d'entre elles correspondent exactement à notre population étudiée : elles sont nées entre 1929 et 1941 , en France, pays où elles ont été cachées et dans lequel elles vivent toujours. II s'agit de dix-sept femmes et de douze hommes. Parmi les cinq autres : trois ne sont pas nées en France. L'une d'entre elles est née en Belgique, en 1935, une deuxième est née aux Pays-Bas en 1938. Elles sont venues en France après la guerre. La troisième personne était adolescente durant la guerre : elle est née en 1924 en Pologne et est arrivée en France en 1934. Quatre autres personnes interrogées sont nées en France en 1924, 1926 et 1928. Les entretiens avec des personnes qui étaient adolescentes pendant la guerre viennent apporter un éclairage différentiel sur le vécu des enfants.

Notre objectif était de récolter du matériel concernant les différentes problématiques en jeu dans cette situation inédite des "enfants cachés", les logiques et les solutions mises en œuvre pour chacune d'entre elles. Ce sont des trajectoires de vie que nous avons recueillies.

Nous avons proposé à chacun de relater les événements qui ont traversé sa vie, par un entretien semi-directif. Les questions posées concernent l'histoire de la famille, et tentent de remonter le plus loin possible dans le temps. L'interrogation porte notamment sur les modes de vie de la famille avant, pendant et après la Shoah, mais aussi sur les informations que chacun possède sur l'histoire de sa famille.

II importait de faire apparaître les différents "mondes" que chacun avait traversés, de retracer l'histoire de la famille en récoltant le plus d'informations sur leurs différents univers: citadin, rural, ashkénaze, sépharade, difficultés liées au changement de famille, de langue, de religion, rupture entre les rituels juifs et les rites chrétiens, les situations de camouflage,... II s'agissait de mettre en évidence les liens avec les personnes, les objets ; le parcours personnel de chacun au sein de l'histoire familiale et collective, de nommer les lieux, les communautés, les actes, les rituels réalisés et les objets utilisés.

\section{Illustration clinique}

Nous avons choisi d'exposer ici une seule situation, de façon détaillée. Cette situation recouvre la plupart des problématiques de vie traversées par les enfants juifs cachés en France pendant la Seconde Guerre mondiale.

Simone est née en 1937 à Paris. Elle a trois sœurs : une sœur aînée, une sœur cadette et une sœur jumelle. Ses parents viennent de Pologne, ils sont originaires de Varsovie. Ils arrivent en France entre 1925 et 1927 . Ils se rencontrent à Paris. Le père de Simone a le statut de réfugié politique, il est maroquinier. Son grand-père paternel était tanneur en Pologne. Avant la guerre, la situation financière est satisfaisante, Simone parle d'une maison de campagne et d'une personne à domicile. De sa mère, elle ne sait rien.

Ses parents parlent trois langues. Simone dit qu'à la maison, ses parents se disputaient en polonais et en russe. Le yiddish est la langue maternelle. Son père a un fort accent yiddish et écrit mal le français. 
Simone découvre les noms de ses grands-parents paternels et maternels en août 2006, en recevant une copie de l'acte de mariage de ses parents. C'est par ce biais qu'elle apprend qu'un cousin, qui était témoin du mariage de ses parents, était aussi à Paris avant la guerre ; elle en retrouve un vague souvenir.

Son père demande la naturalisation française pour ses quatre enfants ainsi que pour lui et sa femme en avril 1937 ; il l'obtient seulement pour ses enfants.

C'est au titre de ses activités politiques que son père est dénoncé et arrêté en 1939. Il est déporté en septembre 1942. II reviendra d'Auschwitz en 1945.

Simone ne sait rien de la période de la guerre. Elle a retrouvé en juillet 2006, via I'O.S.E. ${ }^{4}$, le nom du village dans l'Allier où elle était cachée, ainsi qu'une copie de son certificat de baptême datant d'août 1942. Elle a été, entre 1942 et 1944, cachée par l'O.S.E. : en maison d'enfants tout d'abord, puis à partir de 1943 dans des familles. Elle a aussi appris que sa mère et sa sœur aînée étaient ensemble, assignées à résidence dans le Cher. Elle sait également que de 1945 à 1947, elle était accueillie dans une maison de I'O.P.E.J. ${ }^{5}$ avec sa sœur jumelle et leur cadette, mais elles ignoraient alors qu'elles étaient sœurs.

En novembre 1947, le rabbin de la maison de l'O.P.E.J. annonce avec joie aux trois filles qu'elles vont retrouver leurs parents. Une fête est d'ailleurs organisée à cette occasion. Elle se souvient du plaisir qu'elle a eu à manger du chocolat. Depuis, elle dit en raffoler. Le deuxième souvenir date du moment où elle se retrouve sur le quai de la gare avec ses sœurs et leurs parents. Elle se souvient de son père, transformé physiquement: "Il était très abîmé physiquement, on lui a inoculé un certain nombre de maladies et fait des opérations. II a eu la varicelle, donc il a un visage en passoire et ça se voit. II est très marqué". L'assistante sociale, fière de voir une famille au complet à nouveau réunie, fait une photo et la donne au père de Simone. De retour dans leur appartement à Paris, le père de Simone, dans une grande colère, déchire la photo et la jette à la poubelle. Par ailleurs, alors âgée de quatorze ans, la sœur aînée de Simone rentre en 1944 enceinte, l'enfant est placé à l'O.P.E.J. Elle n'apprend ceci que très récemment.

Dans les années d'après-guerre, Simone prend connaissance du fait que pour envoyer des colis à son père, colis qu'il n'a jamais reçus, sa mère a eu des relations avec des hommes. Le père de Simone ne le supportant pas, il demande rapidement le divorce. De 1947 à 1950, ses parents sont encore ensemble. Ils se disputent souvent et violemment. Le climat va en se dégradant de plus en plus. Leur père est violent avec sa femme mais aussi avec les jumelles. II les frappe souvent sans raison, les gifles et les coups de ceinture sont fréquents.

En 1951, les parents de Simone se séparent. Le père obtient la garde des enfants. Leur mère est déchue de sa fonction maternelle. Dès lors, son père interdit à ses filles d'avoir des contacts avec leur mère. Simone reverra sa mère seulement en 1979, mais elle dit que sa mère ne la reconnaîtra pas.

Le récit que fait Simone de son histoire entre 1937 et 1951 est révélateur de deux éléments fondamentaux. C'est l'attaque continuelle du lien de filiation et du lien d'affiliation, qui se poursuit bien après 1945 avec deux périodes distinctes : 1942 1945 et 1945 - 1951, avec une continuité de ces attaques.

\footnotetext{
${ }^{4}$ OEuvre de Secours aux Enfants. Organisme qui participa au sauvetage de nombreux enfants juifs pendant l'Occupation.

5 CEuvre de Protection de l'Enfance Juive. L'O.P.E.J. dirige après la guerre, quatorze maisons d'enfants, pour la plupart, orphelins de la Shoah.
} 
Première rupture. Entre 1942 et 1945, Simone est séparée de ses parents contraints de la mettre à l'abri. Pendant deux ans, Simone est sous la responsabilité de I'O.S.E., d'abord en maison d'enfants, puis en famille(s) "d'accueil". Une autre rupture a lieu au cours de ces deux années. Simone a retrouvé un certificat de baptême datant d'août 1942. Donc, de la maison d'enfants, elle est passée par une famille ou plusieurs familles "d'accueil". Le danger étant de plus en plus grand, il y a eu nécessité de placer ces enfants dans des familles chrétiennes. Et afin de camoufler ces enfants juifs, l'acte de désaffiliation au groupe juif a résidé en leur conversion au catholicisme ; leur affiliation au catholicisme. Dans le même temps, l'éclatement de la fratrie représente une rupture supplémentaire.

Puis de 1945 à 1947, Simone est sous la responsabilité de l'O.P.E.J., dans une maison d'enfants à Cessieu, dans la banlieue lyonnaise. Simone vit à nouveau en collectivité et dans une maison où les "règles juives" sont respectées (observance des fêtes notamment). Elle y étudie même l'hébreu. Simone apprendra plus tard que les autres enfants, tous orphelins, ont fait leur Alyah ${ }^{6}$ et sont partis vivre en Israël. Seules, elle et ses deux sœurs sont restées en France.

\section{Attaque des liens de filiation et d'affiliation}

La métamorphose de l'identité provoquée par l'attaque de l'interaction des liens de filiation et d'affiliation a été totale dans la mesure où Simone ne découvre qu'à l'âge de dix ans qu'elle a des sœurs, et en particulier sa jumelle.

D'autre part, ces attaques vécues entre 1942 et 1945, et qui sont la base de la traque des Juifs pendant la guerre, se poursuivent à partir de 1947, mais cette fois-ci la menace ne vient plus de l'extérieur mais de l'intérieur. Tout d'abord, dès la reconstitution de la famille en 1947, le père de Simone déchire la photo de famille prise sur le quai le jour des retrouvailles, mettant symboliquement fin à l'existence de sa famille. Puis, il répudie sa femme, mère de ses enfants, et menace ces derniers de rompre avec eux si un lien continue à exister entre eux et leur mère. L'attaque de ces liens vient du père de Simone, qui semble poursuivre le travail "inachevé" par les nazis. II est lui-même dans un processus d'attaque systématique des liens. La guerre psychique continue.

Concernant son judaïsme, Simone a des souvenirs de la période d'après-guerre. Elle se souvient de la cuisine faite le vendredi soir. Elle se souvient d'être allée à la synagogue trois fois quand sa mère était encore là. Elle a dormi parfois sur le balcon, lors de la fête de Souccoth ${ }^{7}$. Mais à l'époque, elle ne sait pas que tous ces rituels sont juifs, car aucune explication n'est donnée, ou elle ne les entend pas. Au vu de la tension qui règne au sein de la famille, Simone pense qu'il s'agit de rituels familiaux et non de rituels liés à un groupe culturel, ce qui révèle l'isolement dans lequel se trouve cette famille. Une inadéquation entre le vécu des rites et la logique qui y est associée prend place au sein même de la famille.

\footnotetext{
${ }^{6}$ En hébreu, signifie "la montée", l'émigration vers Israël.

7 En hébreu, signifie "cabanes". C'est une des trois grandes fêtes de pèlerinage ou fêtes des moissons. Célébrée durant sept jours à partir du 15 Tichri, Soukkoth est la plus joyeuse des fêtes prescrites par le Pentateuque. Les Juifs s'installent dans des cabanes rituelles, qui sont les symboles de la protection divine puisqu'elles rappellent les abris fragiles où les Hébreux vécurent dans le désert.
} 


\section{Les silences continuels}

Simone dira à plusieurs reprises durant notre entretien que rien ne se dit dans sa famille. II semble que la brutalité de son père est associée à des silences pesants : "Mon père est très brutal, il parle très très peu, mais parle très, très peu". Un silence lourd, que Simone qualifie de traumatisant. D'ailleurs, Simone en parle au présent, comme si elle le vivait encore aujourd'hui de cette façon. Elle semble en état d'abréaction.

Silences sur le vécu de chacun des membres de la famille. Silences sur ce que son père a vécu pendant la guerre. Silence sur l'issue du divorce entre ses parents: "Parce qu'on n'a pas su le résultat du divorce, du jugement, on l'a jamais su. Je l'ai découvert trente ans après". Silence sur la nature des relations entre sa sœur aînée et son père. Au départ de leur mère, c'est la sœur aînée de Simone qui assure la logistique familiale. Elle continuera à vivre avec son père jusqu'à son décès. Silences sur la vie de sa mère et de sa sœur aînée pendant la guerre. Silences sur l'enfant que sa sœur aînée a eu en 1944. Elle décrit ce silence de la façon suivante : "Il y a cinq personnes qui sont chacune dans un couloir, et il y a à gérer ce qu'elles sont au fond, ce qu'elles ont vécu au fond, et la réalité d'aujourd'hui avec laquelle il faut faire". Ce silence est semblable au silence du couloir de la mort. II participe à la confusion de ce qui est vivant et de ce qui est mort. II crée un isolement de chacun, une rupture de liens des uns vis-à-vis des autres et de l'extérieur. Ce silence partagé est tel qu'il ressemble à une amnésie familiale, qui perdure aujourd'hui. Simone ne sait pas ce qu'elle a vécu avant l'âge de dix ans.

Les seules façons de communiquer dans la famille, résident dans les passages à l'acte: les gifles, les coups donnés par le père, la fugue de Simone et de sa sœur jumelle.

Le silence n'est pas là pour protéger, il est violent.

Tout ce qui constitue le "noyau" culturel juif de Simone, au sens des ingrédients culturels comme la langue, les rituels, les manières de faire, semble donc éparpillé et perdu. Les peurs dues au fait d'être juive, d'être menacée comme juive, et celles dues aux attitudes de son père ont fait volé en éclat ce "noyau", "noyau" ou sorte de bagage intime, nécessaire, avec lequel chaque être se construit personnellement, en lien avec son groupe familial et culturel.

\section{Les humiliations, les maltraitances}

Simone a subi des humiliations de deux ordres. D'une part en tant que Juive, et d'autre part en tant qu'être humain. Elle ne parle que des humiliations et de la maltraitance qu'elle connaît à partir de 1947.

Tout d'abord, elle subit des humiliations en tant que Juive. Elle raconte notamment son retour à l'école communale en 1947, où son institutrice lui fait sentir qu'elle n'est pas comme les autres, elle la menace même en lui disant : "La guerre n'est pas loin". Elle raconte aussi qu'un jour, elle se promène dans la rue en compagnie de sa sœur jumelle et de son père, une personne les interpelle en les traitant de "youpins". Blessé par cette remarque, le père de Simone crache à la figure de cette personne. Ne comprenant pas le sens du mot "youpin", Simone demande une explication à son père, qui, en réponse, lui donne une paire de gifles. Cette réponse de son père est 
une véritable réitération en actes de l'humiliation. Simone ne peut que subir les projections violentes de son père.

Récemment, Simone s'est fait traiter de sale juive par un garçon de dix ans dans un bus. Elle dit avoir été surprise et ne pas avoir compris comment elle a pu être identifiée comme Juive. L'âge de ce garçon correspond à l'âge de ses premiers souvenirs d'enfance.

Simone est maltraitée par son père. Toute la violence du père de Simone rejaillit systématiquement sur ses filles jumelles. C'est comme si la gémellité incarnait le clivage produit par le traumatisme de chacun des membres de cette famille.

Au sortir de la guerre, Simone et sa sœur jumelle sont rachitiques. Elles sont suivies régulièrement par une assistante sociale des Allocations familiales. Leur père fait tout pour qu'elles prennent du poids, instituant cinq repas par jour. Ainsi à quatorze ans, Simone et sa sœur jumelle atteignent chacune le poids de quatre-vingt-seize kilos. Il considère ses filles comme objet de gavage et contrôle leur corps.

La brutalité de son père est l'élément le plus insupportable pour Simone. Simone se sent libérée de cette violence lors de son hospitalisation pour sa paralysie. Son corps s'est-il figé à ce moment-là ? Elle a passé alors deux ans à l'extérieur de la maison, dont une année en maison de repos.

Par ailleurs, le père de Simone se soumet à l'injonction de l'assistante sociale d'envoyer Simone et sa sœur jumelle en colonies de vacances pendant l'été. Ces moments-là sont décrits par Simone comme des moments de bonheur, de liberté, de découverte. Elle et sa soeur peuvent enfin respirer, en-dehors de la "vie" familiale.

Simone est dénigrée en tant que Juive, du fait de son appartenance à son groupe culturel, dénigrement qu'elle a connu pendant la guerre, puis après la guerre par les insultes venant de l'extérieur, de l'espace extra-familial. Simone est déniée aussi en tant qu'être humain par son père, par celui grâce auquel elle est venue au monde, par celui grâce auquel elle est vivante.

\section{Des conséquences du trauma}

Les événements de vie vécus par Simone sont traumatisants. Nous identifions notamment des troubles d'affiliation, des troubles identitaires et des troubles au niveau familial.

Un processus de désaffiliation et de réaffiliation s'opère par son changement de prénom. Son prénom à l'état civil n'est pas Simone. Ce prénom lui est donné en 1942 au moment de son baptême. Elle "renaît" à l'âge de cinq ans en enfant catholique. Á sept ans, réintégrant une maison juive, à l'O.P.E.J., elle est réaffiliée au monde culturel de ses parents, par les rituels imposés et par son prénom de naissance qu'elle retrouve. Á dix ans, ce sont les retrouvailles familiales, ses parents lui donnent un diminutif mais avec le fort accent yiddish de son père, ce diminutif sonne comme un prénom masculin.

Entre 1947 et 1956, Simone est élevée dans le judaïsme mais sans le savoir : pour elle, les rituels juifs sont des rituels familiaux.

En 1960, elle choisit le catholicisme, à la suite de la rencontre avec des religieuses qui lui prodiguent de l'affection. Elle est baptisée et choisit Simone comme prénom 
de baptême, sans savoir qu'elle a déjà été baptisée et qu'elle a déjà porté ce prénom-là.

Á partir de ce moment-là, elle porte deux prénoms et a deux vies. Elle se clive dans le réel. Son mari, la famille de son mari, ses collègues et amis la connaissent sous le prénom de Simone, ignorant que ce n'est pas son prénom originel. Ses sœurs et son père continuent à l'appeler par son diminutif.

Le clivage qui, de fait, se met en œuvre en 1942 pour protéger cette petite fille âgée de cinq ans, est réactivé à partir de 1960, au moment où Simone dissocie sa vie familiale d'origine de sa vie chrétienne, et de façon encore plus manifeste à partir du moment où elle va commencer à s'occuper de sa mère.

En effet, en 1977, Simone, ainsi que ses sœurs sont convoquées par la préfecture de police. Seule Simone se rend à la convocation lorsqu'elle apprend qu'il s'agit de s'occuper de sa mère. La mère de Simone avait été prise en charge pendant plusieurs années par un couple jusqu'à son départ pour Israël. Á partir de 1979, avec l'aide de sa sœur jumelle, Simone s'occupe de sa mère. Cet appel aux enfants s'inscrit dans le cadre de la loi sur l'obligation alimentaire. Simone va prendre soin de sa mère, jusqu'à sa mort en 1990. Elle dit redécouvrir alors les rituels juifs par l'intermédiaire de sa mère, qu'elle place délibérément dans une maison de retraite juive. Elle est impressionnée par sa mère, qui chante des chansons en yiddish et qui fait l'admiration des autres résidants et du personnel de la maison. En parallèle, elle mène sa vie de chrétienne, en élevant ses filles dans le catholicisme, ne dévoilant même jamais à ses filles qu'elle est juive et en allant régulièrement à l'église. Elle mène une vie dissociée.

Déçue par l'Eglise, elle quitte le christianisme qu'elle juge contradictoire avec son engagement politique. Elle se sépare un peu plus tard de son mari. Et c'est à partir de ces années-là, se rapprochant de sa mère et de ses racines par un travail en psychothérapie jungienne, qu'elle tente de reconstruire son "noyau" culturel juif.

Il y a dix ans, elle rencontre le bouddhisme, qu'elle dit être en complémentarité avec le judaïsme. Elle considère le bouddhisme comme une continuité de son chemin personnel qui va vers l'apaisement.

Aujourd'hui, elle considère que le fait d'être juive constitue une force de vie. Cette considération s'inscrit dans un paradoxe, et c'est la raison pour laquelle on peut parler de troubles d'affiliation, dans la mesure où c'est le fait même d'être juive qui a fait voler en éclats sa vie personnelle et familiale. Le parcours affiliatoire que Simone traverse en tant qu'adulte est un parcours métissé et dissocié qu'elle construit seule. Ce parcours est métissé dans la mesure où Simone utilise des voies multiples.

\section{Des troubles identitaires}

Compte tenu de son parcours traumatique et ses tentatives de s'en sortir, Simone présente des troubles identitaires qui se traduisent par une symptomatologie spécifique.

Simone dit être incomprise. Elle ne se sent jamais à la bonne place. Elle dit avoir fréquemment de fortes angoisses.

Elle souffre de problèmes de poids depuis la guerre : elle est rachitique au sortir de la guerre et devient obèse à l'âge de quatorze ans. Depuis, Simone ne cesse d'avoir des fluctuations de poids. Elle a maigri dès qu'elle est partie de chez son père. L'obésité est probablement un facteur de protection par rapport à son père, mais elle 
dit reprendre du poids à chaque fois qu'elle traverse des périodes difficiles. Simone souffre de paralysies : la première a lieu lorsque sa mère part du domicile familial ; d'autres réapparaissent lorsqu'elle est enceinte. Pendant la durée de ses deux grossesses, Simone reste alitée. Ses deux enfants naissent prématurément à huit mois.

Simone souffre d'une maladie thyroïdienne auto-immune, qui s'est déclarée il y a vingt ans. Elle somatise beaucoup, en ayant des maux de ventre et de la diarrhée. Suite à sa visite au Mémorial de la Shoah à Paris, elle ne veut voir personne, et "s'est vidée" pendant plusieurs jours: "Aucune nourriture ne me tenait, je ne perdais pas de poids, mais pour autant je me vidais. J'avalais un verre d'eau et je sortais un kilo de vilaines choses".

La problématique liée au somatique, au corps, est très importante chez Simone et chez ses parents.

Son père a été soumis à des expériences médicales à Auschwitz. Son corps a été utilisé comme objet à des fins "expérimentales". Par ailleurs, de par ce qu'il a vécu au camp d'Auschwitz, le discernement entre le corps vivant et le corps mort est source de confusion.

Son père, de retour, exprime une forte violence et la met en acte en frappant à la moindre respiration. II attaque la vie. II ne supporte pas les activités ludiques de ses filles jumelles, qui expriment qu'elles sont bien dans la vie.

Sa mère, pour sa survie, a été objet sexuel : femme juive seule pendant la Shoah, n'ayant pas de ressources, elle a utilisé son corps pour subvenir à ses besoins.

En 1947, Simone est maigre, elle doit grossir. Son père procède par gavage. Les enfants gavés deviennent à leur tour objets utiles, corps contrôlés et utilisés par les autres. Le résultat est que le corps de Simone atteint quatre-vingt-seize kilos à la période de l'adolescence. Cette obésité va de pair avec la métamorphose de son corps en période de puberté. Les remaniements psychiques normaux et difficiles à assumer à l'adolescence sont rendus encore plus difficiles par l'atteinte de ce surpoids, mais aussi par l'impossibilité pour Simone de vivre sa période de latence. La socialisation, l'ouverture vers l'extérieur, étaient interdites par un père répressif.

Par ailleurs, s'ajoute à cette surcharge pondérale, la paralysie. Au départ de sa mère, Simone est paralysée, et ces paralysies vont réapparaître au moment de donner la vie, c'est-à-dire quand elle est enceinte.

Dans cette problématique du corps, c'est la vie qui semble menacée.

Les vécus traumatisants du père ont un impact évident sur Simone surtout dans le rapport au corps et à la mort. Simone a aussi un vécu traumatique. Cet ensemble met en avant un discours qui révèle une construction psychique morcelée.

D'abord, les retrouvailles familiales s'apparentent à un morcellement : elles évoquent ces situations d'adoption, où l'enfant se trouve à vivre avec des parents qui se disent être ses parents mais qu'il ne connaît pas. L'enfant est donc face à une situation d'étrangeté/familiarité. Et dans la famille de Simone, les relations "incestuelles" qui s'instaurent semblent tenter de répondre à l'étrangeté par l'intimité.

L'isolement est prégnant chez Simone. Elle dit à deux reprises, à propos des membres de sa famille, en y incluant son ex-mari et ses enfants, qu'ils sont chacun dans un couloir, sans communication.

La rupture, l'isolement, la coupure s'instaurent aussi lorsque Simone, ne pouvant faire face aux besoins débordants de ses filles en bas-âge, préfère "couper", partir conduire sa voiture pendant cent kilomètres et revenir ensuite pour les retrouver de 
façon plus adaptée. On peut aussi penser que la naissance de ses filles a donné lieu à une dépression du post-partum ; c'est à partir de là qu'elle commence un travail en psychothérapie.

Elle se retrouve aujourd'hui dans un profond isolement du fait de l'attitude de sa sœur jumelle, qui, sur un mode défensif, se dissocie de son histoire familiale. On retrouve la dissociation gémellaire. Chacune vit une face de l'histoire familiale.

Dans la façon dont elle raconte son histoire, dans ce vécu dissociatif, on identifie un clivage entre le corps, l'émotion et l'esprit. Sa façon de raconter son histoire dénote aussi un certain mode de "pensée opératoire".

\section{Des troubles au niveau familial}

C'est parce qu'il y a eu un récent malentendu entre elle et sa sœur jumelle que Simone s'est mise à reconstituer son parcours et à écrire un livre. Depuis quelque temps, elle vit difficilement l'éloignement de sa sœur jumelle qui est l'unique personne avec laquelle elle a partagé les moments difficiles de l'enfance et de l'adolescence, et pour laquelle elle a ressenti de la complicité. Sa sœur semble réinventer l'histoire familiale, construisant une néo-réalité. Depuis que Simone retrouve les traces de leur histoire, sa sœur refuse d'y croire. Elle est dans le déni ou dans la dénégation de toutes ces informations que Simone recueille: "Je reçois sur papier, que de 1942 à 1944, nous avons été, donc Monique, Marguerite et moi, dans les différentes maisons de l'O.S.E. et chez des nourrices, et suivent les noms des châteaux mais pas des nourrices, des châteaux : château de Montintin ${ }^{8}$, château de Masglier'. J'envoie la photocopie par Internet à ma sœur. Elle dit: " c'est pas vrai "”. Á cette occasion, Simone apprend par sa nièce que sa sœur jumelle reconstruit l'histoire familiale : elle dit notamment que leur père est un Russe orthodoxe et qu'elle n'est pas juive. Elle pense donc que Simone déforme la réalité et est même délirante par rapport à sa propre histoire.

Simone se retrouve à nouveau isolée de sa famille, d'autant que ses deux filles se sont rapprochées de leur tante, et géographiquement, et affectivement.

Dans les années d'après-guerre, la sœur cadette est brillante à l'école et réussit dans tout ce qu'elle entreprend, elle fait l'admiration de leur père. Simone et sa sœur jumelle ne sont pas aussi performantes que leur sœur. Elles arrêtent l'école et commencent à travailler dans l'atelier du père, sans être payées.

La sœur aînée de Simone prend la fonction de mère, et s'occupe de la logistique familiale aux côtés du père.

Simone semble ne rien avoir jamais partagé avec ses sœurs aînées. Et la façon qu'a leur père de traiter ses filles est inégale. Les filles aînées ne sont pas maltraitées, et font alliance avec le père. La rupture entre les deux aînées et les deux plus petites est réelle et leurs relations n'ont plus évolué depuis que Simone a décidé de s'occuper de sa mère et que, comme il l'avait promis, son père a suspendu toute relation avec elle.

Aujourd'hui, ses trois sœurs sont vivantes. La cadette est mariée, l'aînée vit seule dans l'appartement paternel, et le silence est toujours aussi massif que dans les années d'après-guerre. Tout lien réactivé semble constituer une menace pour chacune.

\footnotetext{
${ }^{8}$ Qui se trouve en Haute-Vienne, proche de Limoges.

${ }^{9}$ Qui se trouve dans la Creuse. Ce château a été fermé en 1943, lors de la dispersion des enfants juifs dans des familles chrétiennes.
} 
Toute l'adolescence de Simone est marquée par la terreur de son père qui a été déporté en 1942 et a survécu à Auschwitz. II semble revenir "métamorphosé" par ce qu'il a vécu. Ce père sort des camps de concentration en y ayant intégré la confusion des logiques, en particulier celle qui dissocie la mort du vivant. Pour Zajde (1993, 1995), les traumatismes des survivants des camps de concentration ont engendré une métamorphose de leur identité qui a des conséquences chez leurs enfants. Elle parle notamment d'un bouleversement du fonctionnement psychique dans le sens de l'appauvrissement et de la destruction des ressources, entraînant une confusion des catégories fondamentales telles que: le vivant / le mort, le Moi / le non-Moi, le dedans / le dehors. "Le survivant n'a pas le support culturel pour reproduire son identique culturellement codé". Quand le survivant se "duplique", c'est son identité négative qui est retrouvée chez son enfant. II transmet son aliénation, son sentiment d'insécurité - et non sa "personnalité ethnique", selon Zajde (1993, 1995). En l'absence de contenant culturel, l'individu est voué à être par lui-même et en luimême le garant de l'événement traumatique ; le fait traumatique perdure intact de génération en génération. Dans son rapport avec son enfant, on constate que le survivant se défait d'une partie de son être. "En l'absence de contenant culturel approprié, le traumatisme et les dépressions qui en découlent sont voués à être transmis aux générations suivantes sur le mode de la répétition. Les enfants s'approprient les traumatismes parentaux comme s'ils les avaient vécus eux-mêmes. Ils deviennent alors les contenants de l'histoire parentale au lieu d'en être les héritiers" (p.125).

Le père revient de déportation et ne tient pas compte du vécu de ses filles, et en particulier des plus jeunes. II tente de reconstruire sa vie matérielle avec les moyens qu'il a. II semble être dans une tentative de maîtrise de chaque acte de la vie de ses enfants, et dénie leurs besoins. Les jumelles deviennent le réceptacle de sa violence. Non seulement le survivant duplique son identité négative, mais en plus il duplique cette négativité sur un enfant qui a déjà lui-même vécu des événements traumatiques. Le père de Simone est dans un déni massif des besoins de ses filles et de ce qu'elles ont vécu avant 1947.

Simone est survivante de la Shoah, "enfant cachée" et enfant de deux parents survivants de la Shoah, dont un des parents est survivant des camps.

Le travail d'élaboration que mène Simone depuis quelques années lui permet de poser des actes. Elle dit s'être rendue, il y a peu de temps, pour la première fois sur la tombe de son père ; elle y a déposé une ceinture en cuir qu'elle a confectionnée elle-même, pour "Iui rendre ses coups". Depuis lors, elle se sent soulagée.

Simone est proche physiquement de sa mère à trois périodes de sa vie, dans la discontinuité. Tout d'abord, entre sa naissance et leur séparation quand elle a cinq ans. De cette période, Simone ne peut rien dire.

Puis la période de 1947 à 1950, Simone a entre dix et treize ans. Les conflits entre ses parents sont violents. Simone se souvient avoir fréquenté la synagogue avec elle, et se souvient de sa façon de cuisiner. II s'agit là d'événements associés à des affects positifs. C'est aussi un sentiment de pitié, ou de compassion, que Simone semble éprouver pour sa mère. Elle est malmenée comme Simone est maltraitée. La mère est déchue de sa fonction maternelle à l'intérieur de la maison par l'attitude du père. Elle ne peut que se soumettre à la violence du père et une confusion générationnelle semble alors s'opérer : Simone et sa mère sont au même niveau. Ou 
peut-être projette-t-elle aussi sa propre tragédie sur celle de sa mère : répudiée par son mari, elle est abandonnée, comme un parent pourrait abandonner son enfant.

Enfin, à partir de 1979 et jusqu'en 1990, Simone s'occupant de sa mère, l'inversion générationnelle a de nouveau lieu: c'est l'enfant abandonnée que Simone va retrouver. C'est un choc pour elle et sa sœur jumelle quand elles revoient leur mère : celle-ci est dans une maison de retraite dans laquelle elle ne reçoit aucun soin, et elle ne les reconnaît pas. "On arrive dans cette maison de retraite et on découvre, moi je crois que j'ai tenu bon parce que ma sœur jumelle faisait presque une crise de nerf, on découvre une petite bonne femme, assise sur une taie d'oreiller, avec une barbe de six mois, qui méthodiquement - mais essayez de le faire, je l'ai fait déchire les vêtements, déchire, ça veut dire quelque chose d'extrêmement fort au niveau de ce geste, elle déchire méthodiquement tout un tas de vêtements qu'elle a été chercher dans les armoires". Simone décrit une femme qui présente une symptomatologie psychotique et qui fait l'objet de maltraitance par l'équipe de la maison de retraite. Cette mère qui a été maltraitée par son mari, continue à l'être à la fin de sa vie par l'institution. Et pourtant, c'est par cette mère, qui n'assume plus son rôle de mère, que Simone va redécouvrir sa filiation, renouer avec la vie, comme une nouvelle naissance. Ces retrouvailles déclenchent un mouvement interne : elles viennent réactiver les séparations, les abandons, que Simone souhaite réparer.

En 1962, Simone se marie avec un homme non-juif, qu'elle rencontre dans le cadre de son engagement militant. Elle épouse un militant, "un autre moi-même". Le mariage a lieu à l'église. Ce mariage est comme une mise en acte de sa métamorphose, de ses nouvelles affiliations : engagement politique et conversion au catholicisme. Le chemin personnel qu'elle va faire, à partir de la naissance de ses filles, puis la réapparition de sa mère dans sa vie, va conduire Simone à divorcer en 1983, puis à quitter le militantisme et le catholicisme.

Les relations de Simone avec ses filles sont inexistantes. Ses deux filles se sont rapprochées de leur tante - la sœur jumelle de Simone. Simone dit n'avoir pas su et ne pas savoir communiquer avec elles. C'est la raison pour laquelle elle a eu besoin d'écrire son histoire : pour faire savoir et transmettre. II y a comme une répétition du schéma familial de la génération précédente.

La relation que Simone entretient avec son neveu et sa nièce est satisfaisante. Ce croisement de liens transgénérationnels laisse supposer que le lien de filiation directe est insupportable et inélaborable, à l'image de la relation que Simone a eue avec son père. Le lien direct est source de négativité. La distance physique et psychique apparaît comme le seul moyen de se protéger.

Pour ce faire, les filles de Simone se rapprochent de leur tante : celle-ci réinvente I'histoire familiale en des termes qui sont pour elles davantage pensables et supportables. Simone ne sait pas si ses filles savent qu'elle est juive. Personne dans la famille, à part sa nièce, n'a lu son récit.

N'ayant pas de lien avec ses filles, Simone n'a pas de relations avec ses petitsenfants. En revanche, elle a de bonnes relations avec les enfants de son neveu et sa nièce.

Des compétences et des lignes d'existence

Malgré un parcours de vie chaotique et douloureux, depuis l'âge de vingt ans, Simone tente de trouver des stratégies d'existence, à la fois par des démarches 
d'appartenance à des groupes, et par un travail d'élaboration individuel. Sa ligne d'existence la plus récente consiste, depuis un an, à réaliser un travail de retour sur le passé, en cherchant à reconstituer son parcours.

La première stratégie d'existence que l'on peut repérer, c'est la fugue qu'elle met en œuvre avec sa sœur jumelle: elles n'ont que dix-neuf ans, alors qu'à l'époque, comme l'a souligné Simone, la majorité était fixée à vingt-et-un ans. Cette fuite est le moyen qu'elles trouvent toutes les deux pour se protéger de la brutalité de leur père, et espérer avoir une vie meilleure ailleurs. Elles décident de fuir l'environnement familial pour se construire. Elles espèrent trouver d'autres modèles identificatoires à ce moment-là. C'est en effet le cas pour Simone: l'engagement politique qu'elle rencontre par l'intermédiaire de son expérience professionnelle, et sa conversion au catholicisme, lorsqu'elle tombe malade. Simone trouve un travail dans une usine comme ouvrière. Son dynamisme et son engagement lui permettent d'être repérée par un contremaître qui l'aide à gravir les échelons. Elle suit une formation en comptabilité, et devient militante ouvrière.

Par ailleurs, Simone tombe à nouveau malade - maladie qu'elle ne nomme pas. Elle est recueillie à ce moment-là par des religieuses qui prennent soin d'elle. Peu de temps après, en 1960, Simone opte pour le catholicisme et se convertit.

Cependant le père de Simone semble rester en arrière-fond un modèle identificatoire, quand elle évoque son militantisme. Durant la période vécue avec son père, pulsions de vie et de mort étaient mêlées. Quand Simone dit : "Je respirais, je recevais une correction à coup de ceinture, je ne respirais pas, je recevais une correction à coups de ceinture", la confusion est totale. Simone parle d'un père en colère, or de façon paradoxale, la colère est une impulsion de vie. Le père de Simone était engagé et défendait des idées politiques ; c'est d'ailleurs la raison pour laquelle il est venu en France. Simone souligne que la seule valeur positive que lui a apportée son père, c'est justement ce militantisme. Or, l'engagement politique signifie l'intégration dans un groupe de personnes qui partagent un certain nombre d'implicites. Le militantisme est ainsi un mécanisme de défense positif, une stratégie d'adaptation, qui signe la victoire des pulsions de vie sur les pulsions de mort. II permet la transformation des objets bruts en des objets de pensée. Ce qui est la manifestation d'une capacité de sublimation.

Le militantisme est une nouvelle famille que Simone rencontre à ce moment-là. Elle rencontre d'ailleurs son mari dans un de ces groupes, et c'est la raison pour laquelle elle définit la nature de leur relation de la façon suivante : "J'ai pas choisi un mari, j'ai choisi un militant". Un groupe partage des valeurs, adhère à un mythe, et chacun est le miroir de l'autre. La rencontre de ce groupe politique est un facteur de "résilience" pour Simone, dans le sens où cette rencontre lui permet de se construire psychiquement.

La deuxième rencontre est l'Eglise. Cette rencontre s'effectue sur un mode affectif. Á l'âge de vingt-deux ans, pour la première fois, des personnes s'intéressent à Simone, prennent soin d'elle. Elle est regardée, une attention est portée sur elle. Ce sont des religieuses. Pour Simone, qui a connu un parcours abandonnique pendant plus de vingt ans, la rencontre avec ces religieuses constitue une étape réparatrice de son existence. L'affection vient de ces religieuses; de fait, Simone s'affilie à elles en se convertissant au catholicisme. 
Simone se marie. Elle parle peu de son mari, mais davantage de sa belle-mère, qu'elle dit appeler "maman". La mère de son conjoint devient alors une mère sublimée, elle la décrit comme une bonne mère, mère de substitution.

Son autre ligne d'existence est la psychothérapie. C'est aussi un choix d'affiliation. Face aux difficultés qu'elle rencontre en tant que mère avec ses deux filles en basâge, Simone fait la démarche d'un soin pour elle. Ses deux grossesses ont été pénibles à vivre, elle a dû rester allongée. Lorsque ses filles étaient bébés, elle sentait qu'elle n'avait pas une attitude maternante, et donc un comportement adapté. La violence de son père, qui était enkystée dans son psychisme, rejaillissait sous forme de pulsions archaïques auprès de ses filles. Elle les confiait alors à sa bellemère et sortait pour revenir plus apaisée. L'analyse, freudienne puis jungienne pendant plusieurs années, l'a aidée à comprendre son fonctionnement psychique et l'articulation entre sa vie d'adulte, en tant que femme et mère, avec sa vie d'enfant.

Cherchant à s'apaiser davantage, elle poursuit tout un cheminement personnel qui la conduit à une approche thérapeutique davantage psycho-corporelle.

Ce parcours lui permet de se réconcilier avec le groupe juif, grâce aux retrouvailles avec sa mère, sa première figure d'attachement. Simone met sa mère dans une maison de retraite juive, maison qui respecte les rites et dans laquelle sa mère se met à nouveau à exister dans sa vie. Les retrouvailles sont aussi une reprise de contact avec la vie et le judaïsme : elle retrouve à la maison de retraite la vie juive qu'elle a elle-même vécue enfant, en collectivité, en maisons d'enfants de l'O.S.E et de l'O.P.E.J. Elles se situent dans deux temps concordants. C'est peut-être donc le passage par d'autres groupes qui permet à Simone de retrouver son groupe culturel d'origine.

De plus, Simone a entendu parler de l'association des "Enfants cachés : 1940-1944", et s'est dite réjouie de retrouver une famille. Elle a fait la démarche de participer à un groupe de paroles, mais a été rapidement déçue par ce qu'elle entendait ; elle s'est sentie différente des autres, et a finalement cessé d'y participer. L'histoire des autres "enfants cachés" ne semblait pas entrer en résonance avec son histoire singulière.

Enfin, Simone rencontre le bouddhisme depuis quelques années, groupe qui ne lui demande pas de faire un choix mais qui, au contraire, concilie la multiplicité avec laquelle Simone s'est construite. C'est ce qui semble lui permettre de rendre compatible les différentes parties de son vécu existentiel, dans un mouvement d'unification.

Le passage par le groupe culturel de sa mère, le groupe juif, lui permet de revenir à elle-même, en écrivant son histoire personnelle et familiale. Elle espère, par cet acte d'écriture, se réinscrire dans son histoire, et surtout transmettre. Ce processus d'écriture signifie aussi être dans un processus de transformation des objets bruts. C'est par là même une capacité à élaborer les éléments du traumatisme vécu pendant son enfance.

Depuis quelque temps, Simone s'est inscrite à des cours d'hébreu, et espère un jour faire un voyage en Israël, quand elle aura "plus de forces", dit-elle.

Son cheminement durant ces dernières années montre que Simone tente de réparer son morcellement en cherchant à faire des liens, des ponts: reconstruire une 
continuité face à la discontinuité de son existence, imbriquée dans la discontinuité de l'Histoire collective des "enfants cachés". Citée par Moro (2002, p.123), une phrase de Serres semble tout à fait correspondre au travail que fait Simone, semblable à cet ouvrier tisserand qui "entrelace, tord, assemble, passe dessus, dessous et renoue, le rationnel et l'irrationnel, le dicible et l'indicible, la communication et l'incommunicable".

\section{Discussion}

Cette illustration clinique est révélatrice du vécu spécifique des "enfants cachés". Les "enfants cachés" ont été des enfants "exposés" (Moro, 1989). De plus, ils ont été exposés à un processus de déculturation. Ils présentent ainsi une symptomatologie spécifique et des troubles familiaux sont identifiés.

\section{Les enfants cachés sont des enfants "exposés” (Moro, 1989)}

Au sens mythologique, exposer un enfant consiste à le livrer à un milieu hostile afin de le mettre à l'épreuve du danger, voire de la mort. Ce concept contient l'idée implicite que le risque hors du commun conduit à un destin qui possède une double polarité : soit l'enfant en sort extrêmement fragilisé, soit au contraire, il acquiert des potentialités exceptionnelles.

Ces enfants ont été triplement exposés

Avant même les persécutions en France, de par leur statut d'enfants de migrants juifs, ils étaient vulnérables. Différents travaux montrent que les enfants de migrants sont vulnérables, ils appartiennent à un groupe à risque. Le premier moment de la vulnérabilité de ces enfants est celui de la phase post-natale où le bébé et la mère doivent s'adapter l'un à l'autre et où la triade se retrouve seule, sans la famille restée au pays.

Moro (2003) nomme ces enfants nés en France des enfants "métis", dans la mesure où leur structuration culturelle et psychique se construit sur un clivage et sur un conflit, dans un contexte d'instabilité et de multiplicité. "Ces mécanismes de clivage et de conflit doivent, dès lors, être considérés comme des déterminants de la vulnérabilité des enfants de migrants" (p.175).

Le métissage est ce produit de la double transmission parentale et sociétale, une transmission complète et parfois violente, avec des ruptures et des conflits (Moro, 2007).

Le premier lieu de la dualité est la maison, la filiation. II y a le monde du dedans, celui des parents et le monde du dehors, que représentera l'école à partir de trois, quatre, cinq ou six ans. Le monde de dedans, c'est celui de la langue yiddish, parfois mêlé à d'autres langues : le français mais aussi le polonais, le russe... Pour d'autres, c'est le judéo-espagnol. C'est aussi pour certains, celui des préoccupations politiques fortes, des pères qui appartiennent au Bund ${ }^{10}$ - comme on peut le supposer pour le père de Simone -, qui sont en rupture avec la religion, de ceux qui prônent le sionisme (mais assez rarement pour ceux qui sont venus en France), ou de ceux qui continuent à respecter certains rites et fêtes (Shabbat, Kippour, Rosh

\footnotetext{
${ }^{10}$ En yiddish, c'est l'Union Générale Juive des Travailleurs, organisation socialiste créée à Vilna en 1897.
} 
Ashana, Souccoth ${ }^{11}$ ), de façon traditionaliste. Le monde du dedans, c'est aussi les habitudes culinaires, comme la préparation de la carpe farcie (gefilte fish), des boulettes à la farine d'azyme (kneidlers), ou du foie de volaille haché et des œufs hachés (gehakté leiber mit tsibalèss).

Pendant les persécutions, les enfants ont été exposés par les ruptures, les privations, les humiliations, parfois la maltraitance. Pendant leur période de clandestinité, outre la rupture avec leur milieu, ces enfants ont parfois été cibles d'humiliations et de maltraitance, ils devaient se taire et faire comme si l'avant n'avait jamais existé, quel que soit l'âge qu'ils avaient.

Après les persécutions, à la Libération, en plus d'avoir été cachés, et ainsi miraculés, ils sont devenus orphelins et/ou enfants de survivants de la Shoah. Á la libération, ils sont à nouveau exposés car ils sont devenus cibles des traumatismes et des deuils non aboutis des adultes qu'ils côtoient d'une part, et d'autre part de la société française toute entière. C'est ce que vit Simone : elle est la cible de la maltraitance de la part de son père.

De plus, les "enfants cachés" ont été exposés à un processus de déculturation.

Dans le phénomène de dé-culturation, il y a deux mouvements: celui qui les a conduit de leur univers familier à un univers étranger qui les fait entrer dans la clandestinité, souvent sur le mode de la frayeur, puis celui qui les mène de cet univers devenu familier à l'univers familier emprunt d'étrangeté, quand les persécutions sont terminées et qu'ils sortent de la clandestinité. Lorsqu'on vient chercher ces enfants qui ont été cachés: ils ne reconnaissent plus leur mère, ils parlent le patois, ils portent un autre prénom, parfois un autre nom, parfois ils adhèrent à une autre religion. En 1947, Simone ne reconnaît pas ses parents et même pas ses sœurs qui sont dans la même maison d'enfants.

La déculturation est en fait une expérience non réussie, non aboutie de l'acculturation, (c'est-à-dire de rencontre entre deux mondes). C'est atteinte narcissique majeure. Dans le processus qui s'est opéré chez ces enfants entre 1942 et 1945, il est question de désaffilier pour réaffilier, mais cette réaffiliation ne s'effectue pas complètement. Ainsi, la personne reste "ouverte".

\section{Les "enfants cachés" présentent une symptomatologie spécifique}

Cette symptomatologie est liée à l'effraction psychique, la survie, aux attaques d'affiliation, aux pertes et aux deuils impossibles. Cette symptomatologie est renforcée par le silence.

L'effraction psychique : anxiété, angoisses, auto-dévalorisation, doutes, changement de personnalité, tristesse, troubles de la mémoire, problèmes psychosomatiques, amnésie, cauchemars, perte de l'orientation spatio-temporelle.

La survie: hypervigilance, troubles du sommeil, rêves à répétition, répression des émotions, clivage, parfois hyperactivité et silence.

\footnotetext{
${ }^{11}$ Fêtes juives.
} 
Les attaques d'affiliation: impression de ne jamais se sentir à sa place, isolement, impression de ne jamais être compris, être en manque de quelque chose.

Des troubles familiaux intra et inter-générationnels sont identifiés.

Ces troubles sont particulièrement manifestes chez Simone.

II y a tout d'abord les difficiles ou même parfois impossibles retrouvailles avec les parents

Enfants et parents ont espéré se revoir et chacun a idéalisé l'autre et les déceptions ont été importantes. Certains parents sont survivants des camps, d'autres ont connu la clandestinité et les humiliations. Ces retrouvailles se sont révélées être impossibles, et a émergé parfois de la violence physique et/ou psychologique. II y a eu un déni du trauma des enfants, et même plus de ce qu'ils ont vécu, du silence qui les a isolés, et dans certains cas des situations de maltraitance car les pulsions de vie des enfants étaient insupportables pour leurs parents.

La fratrie : l'éclatement de ce sous-système familial

Pour des besoins liés à la protection, frères et sœurs ont souvent été cachés dans des lieux différents. Dans les analyses présentées, certains se sont pourtant retrouvés dans une même famille. Ils ont vécu les événements différemment, chacun selon leur âge et les circonstances.

Les effets de la symptomatologie des uns ont amplifié ceux des autres. Les parents ne pouvant plus fonctionner à la mesure de leur fonction parentale, la symptomatologie a été exacerbée. Ces symptômes ont été amplifiés lors des retrouvailles avec la fratrie. Les conflits au sein de la fratrie sont encore d'actualité : chacun a sa version de l'histoire, selon ses propres aménagements défensifs.

Des difficultés de communication avec leurs propres enfants

La plupart des personnes rencontrées expriment des difficultés relationnelles avec leurs enfants. La littérature psychologique, concernant ce sujet, est très importante et souligne les problèmes de communication entre les deux générations. Gampel (2003) invente le concept de "transmission radioactive", Faimberg (2003) parle de "télescopage de générations" emprunt d'identifications inconscientes aliénantes, Waintrater (2004) expose les traits cliniques de la "famille survivante".

Tous les éléments manifestes chez les "enfants cachés" sont révélateurs de la façon avec laquelle ils se sont construits. Ils mettent ainsi à jour des éléments organisateurs de leur psychisme.

\section{Les éléments organisateurs du psychisme}

Quel que soit l'âge de l'enfant au moment des persécutions, il est possible d'identifier des organisateurs similaires du psychisme, chez les "enfants cachés".

\section{La double "double contrainte"}

Les "enfants cachés", menacés, ont été contraints de mettre en place un "dispositif de survie" permettant de faire face à tout type de situation. Ce "dispositif de survie" a continué à fonctionner après la guerre (Feldman, 2006). "Après la guerre, ils ont dû reprendre le cours normal de leur vie et se développer comme des enfants de leur 
âge, en dépit de tous les manques. Mais au plus profond d'eux-mêmes, ils ont continué à vivre traqués dans le ghetto, dans le camp, comme enfant caché" (Gampel 2003, p.122). Par "dispositif de survie", nous désignons un système mis en place par chacun de ces enfants, sous l'impulsion de la nécessité vitale de se métamorphoser pour échapper aux rafles et donc à la mort. L'enfant a traversé différents mondes et a connu différents objets attachés à ces mondes. C'est avec ceux-là que l'enfant a été contraint de se familiariser, et a ainsi continué à se structurer (conversion, pratique du catholicisme, habitudes de vie des Français ruraux...).

Les peurs et les frayeurs multiples, associées à ces nécessaires métamorphoses, constituent les moteurs de fabrication de ce "dispositif de survie". II semble que celuici se soit constitué sous l'impulsion de deux injonctions paradoxales.

En thérapie familiale systémique, l'injonction paradoxale décrit un type de lien particulier entre deux personnes dont l'issue devient pathologique. Bateson (1956) et l'école de Palo Alto définissent l'injonction paradoxale de la manière suivante : c'est une injonction qui intéresse des relations transcontextuelles (individu, famille, groupes sociaux) entre des personnes impliquées dans un enjeu vital (parentsenfants) qui s'adressent des messages logiquement antinomiques ; ces messages empêchent toute prise de décision cohérente dans les contextes où ces échanges surviennent, mais aussi tout dégagement des personnes vitalement impliquées.

La situation de l'injonction paradoxale est présente quand trois éléments sont réunis : deux ou plusieurs personnes sont engagées dans une relation intense, voire vitale. Dans ce contexte, un message est émis. II affirme quelque chose. C'est l'injonction primaire. Ensuite, il affirme quelque chose sur sa propre affirmation, c'est l'injonction secondaire. Mais parce que ces deux affirmations s'excluent, le sens du message est indécidable. Le récepteur est mis dans l'impossibilité de sortir du cadre fixé par le message.

\section{Faire oublier sans oublier}

Pour un "enfant caché", il s'agit d'oublier qu'il est juif et en même temps de ne pas l'oublier : faire oublier aux autres sans l'oublier soi-même. L'enfant doit cacher qu'il est juif mais ne pas l'oublier; il doit faire oublier aux autres qu'il est juif, il est nécessaire que les autres croient qu'il est comme eux.

II doit se fondre dans la masse en changeant de religion, en allant vivre à la campagne, en changeant de nom, de prénom... Or ne pas oublier d'être juif, c'est, pour les garçons, la marque corporelle de la circoncision : comment l'oublier quand la trace est indélébile ? Ou, par exemple, au travers de la (dernière) phrase adressée par certains parents à leurs enfants, au moment de la séparation : "n'oublie pas que tu es un Juif" ("Fargess nicht du bist a yid" - en yiddish).

\section{S'isoler et s'affilier}

La deuxième injonction paradoxale est la suivante : pour vivre, l'enfant juif est isolé de son groupe d'appartenance, de sa famille, de son milieu familier ; il est placé dans un autre environnement. II est amené à s'inscrire dans un nouveau groupe qui lui est étranger mais dont l'appartenance n'est pas menaçante. II peut s'agir, par exemple, du groupe des catholiques, ou bien d'un tout autre groupe que la personne va rencontrer plusieurs années après la guerre. 
L'isolement de ces enfants était tel que parfois ils se trouvaient placés dans un même village, voire dans une même famille, en ignorant que les autres enfants présents étaient juifs ou qu'ils étaient de la même fratrie.

Chacun accepte de s'isoler de son groupe d'appartenance pour s'attacher à d'autres groupes afin d'assurer sa sécurité. II doit accepter cette séparation et tisser de nouveaux liens; il doit se séparer non seulement de ses parents mais aussi de son monde d'origine. Les nouveaux liens qu'il tisse s'inscrivent donc dans une réaffiliation.

\section{Le miracle}

Comment penser le miracle en psychologie ? Le vécu des "enfants cachés" nous contraint à le penser comme un organisateur de leur psychisme. Dans les rencontres réalisées, ce thème revient comme un leitmotiv : "j'ai eu de la chance" d'être en vie, "pourquoi moi ? Comment ce miracle a-t-il pu m'arriver ?". C'est une question à laquelle, à sa façon, chacun tente de répondre. Le dictionnaire Le Robert définit le mot "miracle" ainsi : "Fait extraordinaire où l'on croit reconnaître une intervention divine bienveillante, auquel on confère une signification spirituelle", "chose étonnante et admirable qui se produit contre toute attente ou "chose admirable dont la réalité semble extraordinaire". Le fait admirable, extraordinaire consiste à être en vie, alors que tant d'autres sont morts. Ce miracle est identifiable de deux façons dans les récits. II est souvent repérable par un acte précis: une date, une heure, une circonstance. Á ce moment-là, le miracle s'accomplit en même temps que la frayeur ou une forte peur. Mais il peut s'agir aussi d'un miracle "diffus", par le fait même d'avoir été caché et d'avoir camouflé son identité.

\section{La clé de voûte des organisateurs psychiques : le secret}

Ces récits mettent en évidence une pérennisation de la vie clandestine dans le fait de continuer à ne pas révéler qui ils sont et ce qu'ils ont vécu. Ils gardent un secret : ne pas dire qu'on est juif, vouloir faire sa communion, aller au catéchisme, continuer à garder son prénom et même parfois son nom d'"enfant caché" ou même franciser son nom ou le changer (Lapierre, 1995), ne pas circoncire ses enfants nés après la guerre ou les circoncire sans intention culturelle ou religieuse, c'est-à-dire invoquer une tout autre raison dans le fait de le réaliser. Dire ou ne pas dire : ces entretiens de recherche montrent que ce fonctionnement est toujours d'actualité. S'adressant au chercheur que j'incarne (Feldman), un témoin me dit, au bout de vingt minutes d'entretien: "Je vous confierais peut-être quelque chose tout à l'heure, une chose qui est sous le sceau du secret." II avouera qu'à l'âge de quarante ans, il était "en manque de quelque chose" en quête de réponses à ses questions, il me parlera de sa rencontre avec la franc-maçonnerie. Ceci me conduit à formuler que ce "dispositif de survie", dont la clé de voûte est le secret, est toujours actif aujourd'hui.

Le secret est fabriqué par le silence, le "double silencié" : extérieur et intérieur, ou la "conspiration du silence".

Á "secret", Vigouroux (1993) donne la définition suivante : "Le mot secret possède deux sens. Il désigne d'abord ce qui reste séparé, retiré, mis à part, dissimulé, impénétrable, environné de mystère. II désigne également un moyen, un mécanisme ingénieux - un mode de connaissance qui permet d'atteindre un certain but" (p.111). Dans cette définition, nous pourrions remplacer le terme "secret" par "enfant juif qui a dû se cacher pour échapper à la mort". La définition serait aussi juste. L'enfant juif 
pendant la guerre a été d'une part, "séparé, mis à part..." et, d'autre part, c'est par lui-même que nous pouvons comprendre l'histoire familiale, il est lui-même, "le mode de connaissance".

C'est le secret qui a sauvé l'enfant et en même temps, c'est peut-être l'élucidation de ce secret qui permettrait de comprendre la fabrication de ce "dispositif de survie", encore actif aujourd'hui. Ici, il ne s'agirait pas d'un secret de famille, dont l'objet secret est dissocié de la personne, mais du "secret-personne" : la personne n'est pas simplement dans le secret, elle est ce secret.

\section{Propositions de soins}

Á partir de ces analyses, on peut faire quelques propositions thérapeutiques au titre de pistes de travail. Aujourd'hui, ces personnes sont âgées, il est donc parfois difficile pour elles de ramener le passé dans le présent et de réaliser un travail d'élaboration, du fait de leur âge. Les pistes proposées constituent quelques réflexions qui seront à approfondir dans un cadre plus large de prise en charge d'enfants, qui vivent actuellement (ou seront amenés à vivre) des situations similaires. II s'agit donc essentiellement de recommandations.

C'est peut-être en cela que la terminologie "enfants cachés", utilisée dans ce document pour parler de personnes qui sont aujourd'hui dans leur dernier cycle de vie, pourrait devenir un concept en psychologie. Ce concept pourrait désigner la situation des enfants qui, pour échapper à un risque mortel, sont contraints de se soustraire de la scène publique et de se cacher. Or, loin d'être uniquement liée à l'époque de la Seconde Guerre mondiale, c'est une situation que traversent actuellement les enfants dont les parents sont sans papiers et menacés d'expulsion.

Étant donné l'absence de prise en considération de ces enfants juifs au sortir de la guerre et pendant de longues années, et la récente reconnaissance de leur souffrance (1991), on ne peut que continuer à les nommer "enfants cachés". Si les quatre recommandations présentées ci-dessous sont suivies, alors il n'y aura peutêtre plus de raisons pour continuer à les nommer "enfants cachés".

\section{Une bonne connaissance des événements historiques}

Aider les "enfants cachés" à élaborer autour des éléments traumatiques impose une connaissance des événements historiques qui ont participé à leur construction psychique. II s'agira alors de reconstituer le parcours du traumatisé. La connaissance des faits historiques, des mouvements de résistance, des déplacements géographiques, des actes mis en œuvre, ainsi que des éléments sociologiques et anthropologiques concernant la population juive, est indispensable pour que puisse se créer une alliance et que le psychologue devienne un véritable partenaire thérapeutique.

Étant donné le phénomène de déculturation vécu par les "enfants cachés", deux intentions thérapeutiques sont à mettre à l'œuvre. Du fait de ce vécu singulier, l'"être juif" originel de la personne semble avoir été dissimulé. Dans un processus thérapeutique, sa révélation permettrait de mettre à jour la façon dont l'individu s'est constitué au cours de ces événements traumatiques. Ainsi, d'un point de vue clinique, peut-être s'agit-il ici, de les aider à sortir de leur cachette, de les "dé-cacher" 
aujourd'hui, pour qu'ils puissent vivre (enfin) sereinement. D'autre part, en réponse au phénomène de déculturation, chacun à sa manière tend à réaliser un "bricolage de métissages". Cette tentative semble parfois vouée à l'échec, tant elle est antagoniste. Une des intentions thérapeutiques sera d'aider les sujets à rendre compatible leur multiplicité culturelle.

\section{L'importance du groupe : rompre l'isolement}

Les différents récits ont montré que l'isolement a été un facteur de renforcement de l'impact traumatique. Ces enfants juifs cachés pendant la guerre ont été en "latence d'identité" pendant quarante-sept ans, jusqu'en 1991, date à laquelle le premier rassemblement des "enfants cachés" pendant la guerre les a officiellement sortis du silence. Le groupe est fondamental dans l'existence d'un individu. La deuxième recommandation consiste donc à soutenir la démarche de l'attachement au groupe, à la collectivité. Le but est de rompre l'isolement en permettant que la parole puisse circuler et que des actes puissent être posés.

Accompagner un sujet dans une telle démarche, c'est aussi l'aider à rendre explicite l'influence du collectif sur l'individu, en identifiant l'ensemble des ingrédients qui ont participé à sa construction.

La génération des "enfants cachés" est aussi appelée la "deuxième génération" - par rapport à celle de leurs parents morts dans les camps ou ayant survécu à la Shoah, faisant du génocide un temps premier. Cette "deuxième génération" est une génération sacrifiée dans la mesure où chacun a été isolé et a tenté, par ses propres moyens, de s'inscrire dans un devenir : avoir une famille et être intégré socialement et professionnellement. En même temps, il s'agit d'une génération qui par cette inscription permet l'accès à la vie, car il s'agit de miraculés ayant donné la vie : ils ont eu des enfants. On peut donc penser que seule la génération suivante - dite "la troisième" : les enfants d'"enfants cachés" - peut offrir du vivant.

Car les miraculés représentent la génération qui était désignée pour morte mais qui a survécu. Malgré l'intention mortelle, alors intériorisée, donner la vie après eux s'est révélé être possible. La génération des "enfants cachés" est celle qui a été témoin de quelque chose qu'il fallait totalement faire disparaître, d'où le silence pesant qui se perpétue aujourd'hui. Les "enfants cachés" ont été la cible d'une déculturation qui les a conduits à chercher un nouveau groupe ou des groupes. Les procédures de réaccueil dans le groupe juif initial, après la guerre, n'ont pas eu lieu. Ils ont donc acquis un statut qui les a rendus étrangers à leur propre groupe: ils sont juifs et autre chose. Or le fait même qu'ils soient juifs et qu'ils aient, en eux, des ingrédients d'autres groupes, ne leur permet pas d'être ou de se sentir juifs à part entière. Ils deviennent davantage des vecteurs du judaïsme. II est alors possible de penser que la génération sacrifiée a pour fonction de transmettre et de faire perdurer le groupe. Intégrer un tel sens dans la construction du récit permet à cette deuxième génération de se réaffilier à la chaîne générationnelle: ils sont enfants de leurs parents, et parents de leurs enfants. Le but est de réintégrer leur récit au sein de l'histoire de leur propre lignée. Même s'il a brûlé dans un incendie, leur arbre généalogique peut se reconstruire. 
La théorie du traumatisme a été construite en psychanalyse à partir de la théorie du sujet. De ce fait, elle contient un point aveugle: elle ne prend pas en compte l'influence et l'action d'un tiers comme étant à l'origine de la souffrance psychique du patient. C'est donc à partir du présupposé initial de l'existence d'un appareil psychique situé dans le sujet que la théorie psychanalytique fonde son modèle de compréhension du trauma. Tout se passe sur la scène intra-psychique.

Sironi (1999) tend vers l'idée d'une modification radicale et novatrice de la théorie du trauma en passant d'une théorie générale du traumatisme aux théories locales du traumatisme. II n'existe aucun système thérapeutique traditionnel qui n'inclut pas la frayeur dans son arsenal étiologique. Dans différents systèmes thérapeutiques à travers le monde, la transformation profonde de l'individu est attribuée tantôt à l'action des sorciers, tantôt à une possession ou encore à la manifestation des malmorts. Dans ce cadre, il me semble que, dans le dispositif de prise en charge des "enfants cachés", l'intentionnalité à l'œuvre dans leur vécu et qui parfois reste encore active aujourd'hui doit être intégrée.

Il est fondamental, et c'est la troisième recommandation, de prendre en compte l'intersubjectif et le collectif. Penser le tiers, l'intention de l'agresseur, est un moyen d'atténuer le "sentiment de culpabilité du survivant".

\section{Le "miracle" au centre du dispositif : une nécessité de la transformation}

Enfin, le "miracle" est au cœur même du vécu des "enfants cachés". L'intégrer dans la construction du récit de vie de chacun constitue la dernière recommandation.

Le "miracle" contraint à la transformation afin d'y donner un sens. L'absence de sens est l'impasse. Ce miracle n'aurait-il pas la fonction de se transformer alors en mission : mission de trans-mission aux générations suivantes ? Le miracle, au cœur de la logique du récit transmis, est investi d'un sens, puisqu'il reprend ainsi un des préceptes du judaïsme : "Tu raconteras à tes enfants".

Si tous ces éléments étaient ainsi considérés dans un dispositif thérapeutique, alors peut-être que la personne ne se définirait plus comme "enfant caché" mais comme un individu ayant vécu une histoire singulière et pour laquelle le récit est écrit et transmis.

\section{Conclusion}

Cette étude constitue un apport pour la psychiatrie humanitaire. Cette histoire ainsi pensée nous permet d'envisager de nouvelles perspectives dans le cadre du soin.

L'individu, qu'il soit enfant ou adulte recourt à de multiples stratégies et comportement, parfois symptomatiques, pour tenter de trouver une sécurité affective, particulièrement si celle-ci a fait défaut dans les premiers moments de l'existence.

On pourrait dire la même chose de cette recherche quasi-pulsionnelle, lorsqu'il y a eu effraction et perte de l'enveloppe culturelle. Ces personnes ont fait le parcours d'une recherche de sens à des phénomènes qui paraissent illogiques tels que tous 
les événements qu'ils ont vécu. Les groupes ont des théories sur les événements et jouent un rôle de réassurance en constituant ainsi un étayage.

Pour rompre le double isolement dans lequel chacun s'est retrouvé, chacun a tenté de trouver des logiques à son existence, souvent en cherchant à appartenir à un groupe.

Ce qui est partagé par l'ensemble des personnes rencontrées, c'est la recherche toujours renouvelée d'appartenance à un groupe. Plusieurs ont rencontré la francmaçonnerie, l'Eglise évangéliste, le bouddhisme ou des groupes de prières, d'autres sont restés attachés au catholicisme, le choix de ces affiliations est entendu comme un chemin évident de reconstruction. II est question d'une tentative d'autoengendrement de la personne elle-même, tout en gardant un attachement au judaïsme, souvent difficile à matérialiser et parfois selon un mode conflictuel. Dans la mesure où les "enfants cachés" sont déculturés, qu'ils ont donc vécu une initiation qui n'a pas abouti, ils adhèrent à ces groupes dans l'intention de chercher à refermer ce processus initiatique resté "ouvert", en suspens.

Cette étude montre l'importance du groupe : la recherche d'étayage est la démarche que presque la plupart des "enfants cachés" ont tenté d'opérer depuis des années, aboutissant parfois à la mise en place d'attachements antagonistes.

La reconnaissance de la souffrance des "enfants cachés" en 1991 a été fondamentale pour toutes ces personnes.

De par un processus d'identification, le collectif fait écho au vécu singulier. La part collective est aussi la part la plus intime. Quand on s'adresse à un individu, c'est en fait au groupe entier à travers l'individu auquel on s'adresse. 